\title{
Fitness cost associated with the chromosomal integron In70.2 in Pseudomonas aeruginosa clinical isolates
}

\author{
Lucia Corich, Linda Furlanis, Lucilla Dolzani, Raffaela Bressan, Enrico Angelo Tonin, Cristina Lagatolla \\ Department of Life Sciences, University of Trieste
}

Key words: Pseudomonas aeruginosa, Integron, Fitness, Outbreak

Costo biologico dell'integrone In70.2 in isolati clinici di Pseudomonas aeruginosa

\section{SUMMARY}

An epidemiologic survey performed at the Trieste University Hospital (northeastern Italy) between 1999 and 2002 revealed a remarkable spread of an MDR Pseudomonas aeruginosa strain, named TS-832035, which carried the chromosomal integron In70.2 containing four gene cassettes (blavim-I, aacA4, aphAl5 and aadAI) in its variable region and conferring resistance to B-lactams, including carbapenems, and to several aminoglycosides. Moreover, some other P. aeruginosa isolates, strictly related to TS-832035 but lacking in the integron In70.2, were detected, but they remained a minor component within the cluster during the three years of surveillance. They showed an MDR phenotype like TS832035 , differing only for the susceptibility level to carbapenems.

The genomic relatedness between TS-832035 and TS-103 was investigated by random amplification of polymorphic DNA (RAPD) typing, pulsed-field gel electrophoresis (PFGE) analysis of Spel-digested genomic DNA, and multilocus sequence typing (MLST). The cost of the integron In70.2 on the fitness of TS- 832035 was determined by performing growth kinetics and direct competition assays against the clonal isolate TS- 103 in three media differing for nutrient availability: a rich medium (Luria Bertani (LB) Broth) and a minimal medium ( $28 \mathrm{~g} / \mathrm{l} \mathrm{K}_{2} \mathrm{HPO}_{4}$, $\left.12 \mathrm{~g} / \mathrm{l} \mathrm{KH} 2 \mathrm{PO}_{4}, 0.4 \mathrm{~g} / \mathrm{l} \mathrm{MgSO}, 7 \mathrm{H}_{2} \mathrm{O}, 4 \mathrm{~g} / \mathrm{l}\left(\mathrm{NH}_{4}\right)_{2} \mathrm{SO}_{4}\right)$ added with a rich carbon source $(0.4 \% \mathrm{w} / \mathrm{v}$ glucose) or with a poorer carbon source $\left(0.4 \% \mathrm{w} / \mathrm{v}\right.$ sodium acetate). Growth kinetic data were obtained by measuring optical density at $600 \mathrm{~nm}\left(\mathrm{OD}_{600}\right)$. For competition assays, the number of $\mathrm{CFU} / \mathrm{ml}$ of each isolate was estimated by colony-hybridization.

We proved the clonality of the two isolates by molecular investigations. The results of the growth kinetics showed the existence of a significant in vitro fitness cost associated with the integron In70.2, more evident in a poorer medium. The sensitivity of the two isolates to the antimicrobial agents tested was the same, except for the different levels of resistance to carbapenems (MIC $16 \mu \mathrm{g} / \mathrm{ml}$ versus $64-128 \mu \mathrm{g} / \mathrm{ml}$ ).

Although we can not exclude that other factors may have favoured the in vivo spread of TS-832035, our results suggest that the increased level of resistance to carbapenems has conferred on this isolate a selective advantage able to compensate metabolic cost associated to the integron.

Un'indagine epidemiologica effettuata nel periodo 19992002 presso l'Ospedale di Trieste ha evidenziato una consistente diffusione di un isolato multiresistente di P. aeruginosa, nominato TS-832035, portatore di un integrone di classe 1, In70.2, la cui porzione variabile contiene 4 cassette geniche (blaVIM-1, aacA4, aphA15 e aadA1) che conferiscono resistenza ai ß-lattamici, compresi i carbapenemi, e a diversi aminoglicosidi $(4,6)$. Al medesimo cluster appartenevano anche alcuni isolati strettamente correlati a TS-832035 ma privi di In70.2, che rimanevano però una componente irrisoria. Essi mostravano un fenotipo di resistenza simile a quello di TS-832035, dal quale differivano solo per il livello di sensibilità ai carbapenemi. Le minime concentrazioni inibenti (MIC) degli isolati privi di In70.2 nei confronti dei carbapenemici erano infatti inferiori a quelle mostrate dagli isolati portatori della cassetta blaVIM-1, sebbene uguali o maggiori ai valori soglia (breakpoints) utilizzati in clinica per identificare i ceppi resistenti. Questo fenotipo è conseguente alla mancata espressione della proteina $\operatorname{OprD}$ da parte dell'intera linea clonale $(4,5)$. Scopo del presente lavoro è stato quello di confrontare i due isolati TS-832035, portatore di In70.2, e TS-103, appartenente al cluster ma privo di In70.2, al fine di valutare il peso metabolico associato all'integrone.

Preliminarmente è stata dimostrata la clonalità di TS-832035 e TS-103 mediante RAPD (Random Amplified Polymorphic DNA)-analysis, macrorestrizione con l'enzima Spe I (5) e MLST (Multilocus Sequence Typing) (2, 3). Successivamente sono state confrontate le curve di crescita dei due ceppi in un terreno ricco (Luria Bertani (LB)) e in un terreno minimo (28 g/l K2 $\mathrm{HPO}_{4}, 12$ g/l $\mathrm{KH}_{2} \mathrm{PO}_{4}, 0.4$ g/l $\mathrm{MgSO}_{4} 7$ g/l H $\mathrm{H}_{2} \mathrm{O}, 4 \mathrm{~g} / \mathrm{l}$ $\left.\left(\mathrm{NH}_{4}\right)_{2} \mathrm{SO}_{4}\right)$, addizionato con una fonte ricca di carbonio $(0.4 \%$ $\mathrm{w} / \mathrm{v}$ di glucosio) o povera $(0.4 \% \mathrm{w} / \mathrm{v}$ di sodio acetato $)$, sia separatamente che in esperimenti di competizione.

L'andamento di crescita delle colture singole è stato seguito mediante misurazione della densità ottica (OD600).

La capacità di crescita in competizione è stata valutata mediante semina su piastra seguita da ibridazione in situ con sonda in grado di rilevare In70.2 (5). Il numero di generazioni $(G)$ è stato calcolato mediante la formula $(\log B$ $\log A) / \log 2$, dove $A$ e $B$ sono, rispettivamente, i numeri di CFU/ml all'inizio del periodo di coltura e alla fine della fase esponenziale (1). Il fitness relativo di TS-832035 è stato calcolato dalla formula GTS-832035 / GTS-103. Le differenze evidenziate dai due isolati nelle cinetiche di crescita e nel numero di generazioni sono state analizzate mediante Test $\mathrm{t}$ di Student; un valore di $p<0.05$ è stato considerato statisticamente significativo.

Il confronto tra le curve di crescita di TS-832035 e TS-103 ha mostrato un diverso effetto del terreno di coltura sulla fitness dei due isolati (Figura I). In LB (Figura IA), non si sono osservate differenze, mentre in terreno minimo addizionato di glucosio (Figura IB), TS-832035 mostrava valori di OD600 2 o 3 volte inferiori a TS-103 $(p<0.05)$. Questo effetto era ancora più evidente in terreno minimo addizionato con sodio acetato (Figura 1C) dove TS-832035 mostrava valori di $\mathrm{OD}_{600}$ anche 8 volte inferiori a quelli di TS-103 $(p<0.01)$. Anche il tempo impiegato dai due isolati per raggiungere la fine della fase esponenziale era dipendente dal mezzo di coltura (Tabella 1 e 2) e la crescita di TS-832035 era particolarmente limitata nel terreno più povero (Tabella 1$)$. Le prove di competizione mostravano un più basso numero di CFU/ml di TS-832035 $(p<0.05)$ durante tutto il periodo di crescita e in ciascuna coltura (Figura II), con differenze più significative nel terreno più povero (Figura IIC) rispetto ai terreni più ricchi (Figure IIA e IIB). Ciò risultava in un numero inferiore di generazioni formate da TS-832035 alla fine della fase esponenziale e in un fitness relativo minore di 1 in tutti i terreni utilizzati (Tabella 2). D’altra parte, la sensibilità dei due isolati agli agenti antimicrobici risultava identica, fatta eccezione per i diversi livelli di resistenza ai carbapenemici (MIC 16 $\mu \mathrm{g} / \mathrm{ml}$ contro $64-128 \mu \mathrm{g} / \mathrm{ml}$ ).

I risultati hanno dimostrato l'esistenza di un significativo

\section{Corresponding author: Corich Lucia}

Università degli Studi di Trieste - Dipartimento di Scienze della Vita

34I00 Trieste - Via L. Giorgieri, I - Tel.: 0405583693 - Fax: 040 558369।

E-mail: luxcor@yahoo.it 
costo metabolico conferito da In70.2 a TS-832035, maggiormente evidente nei mezzi più poveri di nutrienti. Tuttavia, in vivo tale isolato prevaleva nettamente sulla sua controparte mancante di integrone. Sebbene non si possa escludere che altri fattori possano aver favorito l'espansione di TS-832035, i nostri risultati suggeriscono che il maggiore livello di resistenza ai carbapenemici abbia fornito a tale isolato un vantaggio selettivo in grado di compensare il costo metabolico associato all'integrone, anche nei confronti di isolati comunque resistenti secondo i parametri della pratica clinica.

Tabella I. Valori di OD ${ }^{600}$ diTS-832035 eTS- 103 alla fine della fase esponenziale. aRisultati espressi come media $O D^{600} \pm S D$ di 6 esperimenti. ${ }^{b}$ Minimal medium, ${ }^{c} p<0.04,{ }^{d} p<0.03$

\begin{tabular}{|c|c|c|c|c|c|c|}
\hline \multirow{3}{*}{ Isolati di $P$. aeruginosa } & \multicolumn{4}{|c|}{ Fine fase esponenziale ${ }^{a}$} & \multirow{2}{*}{\multicolumn{2}{|c|}{ MM $^{\mathrm{b}}+$ Sodio Acetato }} \\
\hline & \multicolumn{2}{|c|}{ LB } & \multicolumn{2}{|c|}{$M^{b}+$ Glucosio } & & \\
\hline & $\overline{t(h)}$ & $\mathrm{OD}_{600}$ & t (h) & $\mathrm{OD}_{600}$ & t (h) & $\mathrm{OD}_{600}$ \\
\hline TS-832035 & 14 & $2.53 \pm 0.1$ & 34 & $0.88 \pm 0.07^{c}$ & 33 & $0.57 \pm 0.04$ \\
\hline$\overline{T S-103}$ & 14 & $2.57 \pm 0.08$ & 34 & $1.15 \pm 0.08$ & 28 & $0.89 \pm 0.03$ \\
\hline
\end{tabular}

Tabella 2. Fitness relativo (GTS-832035/GTS-103) alla fine della fase esponenziale. aNumero di generazioni, espresso come media \pm SD di 3 esperimenti indipendenti. bMinimal medium. $c p<0.04, d p<0.02$, ep $<0.03$

\begin{tabular}{lcccc}
\hline Medium & Fine fase esponenziale $(\mathrm{h})$ & \multicolumn{2}{c}{ No. generazioni $\left(\mathrm{G}^{\mathrm{a}}\right)$} & Fitness relativo \\
\cline { 2 - 5 } & & $\mathrm{TS}-832035$ & $\mathrm{TS}-103$ & $0.90^{\mathrm{c}}$ \\
\hline $\mathrm{LB}$ & 12 & $17.39 \pm 0.3$ & $19.23 \pm 0.5$ & $0.92^{\mathrm{d}}$ \\
\hline $\mathrm{MM}^{\mathrm{b}}+$ Glucosio & 32 & $17.71 \pm 0.4$ & $19.28 \pm 0.6$ & $0.89^{\mathrm{e}}$ \\
\hline $\mathrm{MM}^{\mathrm{b}}+$ Sodio Acetato & 32 & $16.32 \pm 0.3$ & $18.23 \pm 0.5$ & \\
\hline
\end{tabular}

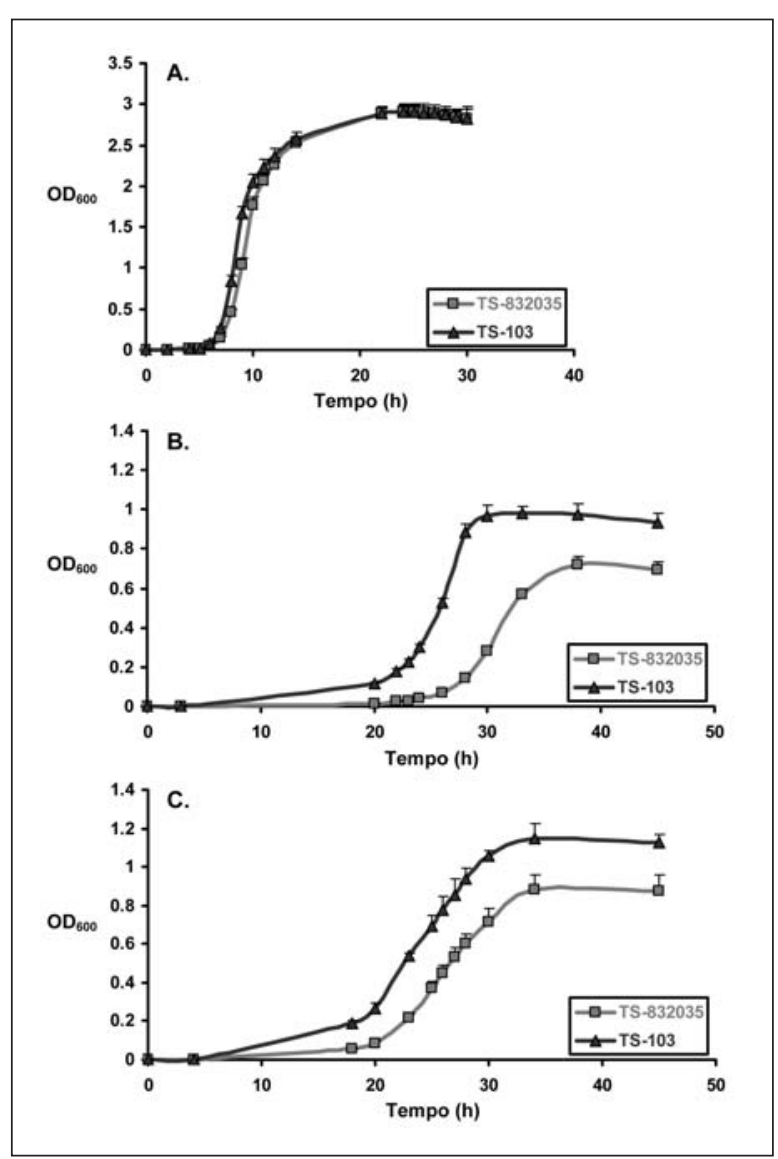

Figura I. Curve di crescita di TS-832035 e TS-103 in LB (A), in terreno minimo + Glucosio $(0.4 \% \mathrm{w} / \mathrm{v})(\mathrm{B})$ e in terreno minimo + Sodio Acetato $(0.4 \% \mathrm{w} / \mathrm{v})(\mathrm{C})$. Risultati espressi come media \pm SD di almeno 6 esperimenti eseguiti con colture indipendenti.

\section{BIBLIOGRAFIA}

1. Billington OJ, et al. Physiological cost of rifampin resistance induced in vitro in Mycobacterium tubercolosis. Antimirob Agents Chemother 1999; 43: 1866-9.

2. Curran B, et al. Development of a multilocus sequence typing scheme for the opportunistic pathogen Pseudomonas aeruginosa. J Clin Microbiol 2004; 42: 5644-9.

3. Giske CG, et al. Estabilishing clonal relationships between VIM-1-like metallo-beta-lactamase-producing Pseudomonas aeruginosa strains from four European countries by multilocus sequence typing. J Clin Microbiol
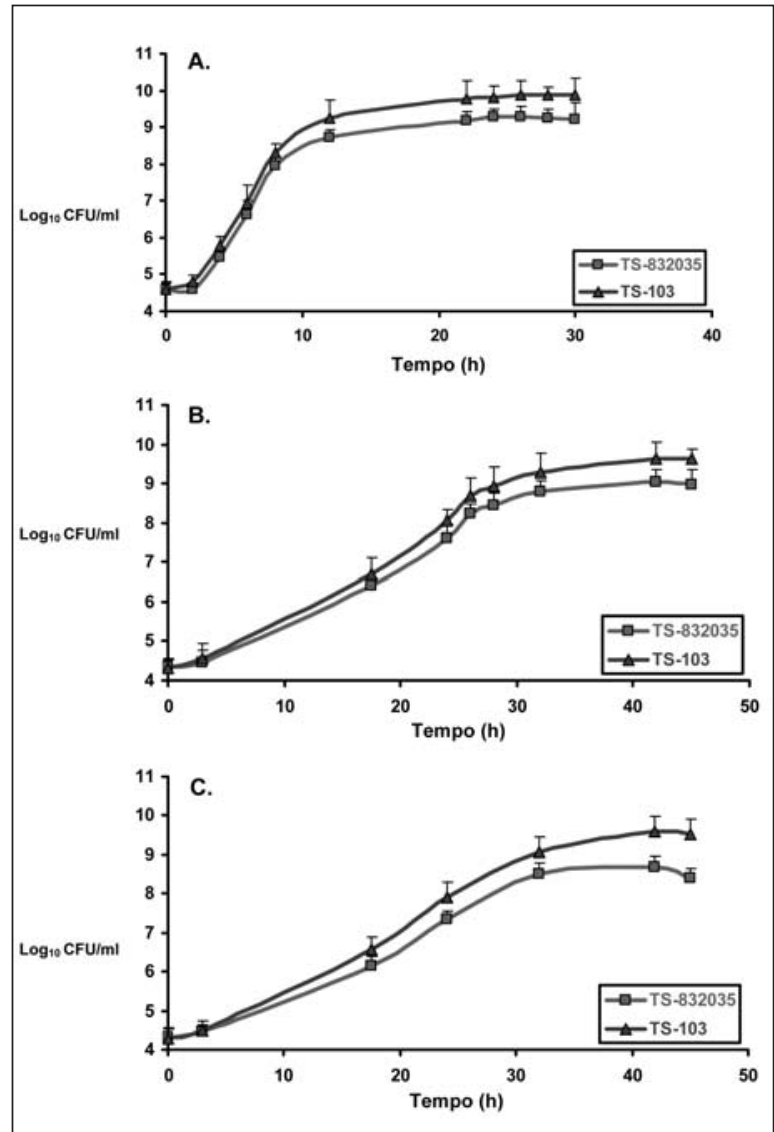

Figura II. Capacità di crescita diTS-832035 in competizione con TS- 103 in $L B(A)$, in terreno minimo + Glucosio $(0.4 \% w / v)(B)$ e in terreno minimo + Sodio Acetato $(0.4 \% \mathrm{w} / \mathrm{v})$ (C). Risultati espressi come media $\pm S D$ di 3 esperimenti eseguiti con colture indipendenti.

2006; 44: 4309-15.

4. Lagatolla C, et al. Endemic carbapenem-resistant Pseudomonas aeruginosa with acquired metallo-beta-lactamase determinants in European hospital. Emerg Infect Dis 2004; 10: 535-8.

5. Lagatolla C, et al. Molecular evolution of metallo-beta-lactamase-producing Pseudomonas aeruginosa in a nosocomial setting of high-level endemicity. J Clin Microbiol 2006; 44: 2348-53.

6. Riccio ML, et al. Clonal relatedness and conserved integron structures in epidemiologically unrelated Pseudomonas aeruginosa strains producing the VIM-1-metallo-beta-lactamase from different Italian hospitals. Antimicrob Agents Chemother 2005; 49: 104-10. 